\title{
Caracterización de la geoforma submarina Galera durante el proceso de extensión de plataforma continental ecuatoriana
}

\author{
Nilton Sánchez, Lourdes Muñoz, Karla Tituana, Isabel García-Arévalo, Carolina Rivas, Andrea Suárez \\ Grupo de Extensión de la Plataforma Continental/Dirección de Plataforma Continental y Fondo Oceánico \\ (DIPAFO)/Instituto Oceanográfico de la Armada (INOCAR) \\ nilton.sanchez@inocar.mil.ec, lourdes.munoz@inocar.mil.ec, karla.tituana@inocar.mil.ec, isabel.garcia@inocar.mil.ec, \\ carolina.rivas@inocar.mil.ec, andrea.suarez@inocar.mil.ec
}

\begin{abstract}
Resumen-El fondo marino presenta un sin número de rasgos submarinos que forman parte de la estructura de la corteza oceánica. Dichos rasgos se nombran en múltiples estudios y publicaciones de acuerdo al nombre otorgado por cada investigador. Sin embargo, a medida que se extiende el estudio del fondo marino es necesario asignarles un nombre mediante consideraciones técnicas que actualmente son estandarizadas por la Organización Hidrográfica Internacional y el Subcomité de los Nombres de las Formas del Relieve Submarino de la GEBCO para la temática de la toponimia submarina. En el presente trabajo, a través del modelado de la data batimétrica con la herramienta en Sistemas de Información Geográfica “ArcGis", se encuentra la determinación de la existencia del monte submarino Galera, que fue aprobado por la SCUFN en 2017 por presentar una elevación de más de $1000 \mathrm{~m}(1027)$ con respecto al relieve circundante. El comportamiento geodinámico del monte Galera es de importancia regional porque se estima que en 15000 años podría encontrarse en el límite de la fosa.
\end{abstract}

Palabras Claves - Asperidades, batimetría, fondo oceánico, montes submarinos

\begin{abstract}
The seabed has a variety of underwater features that conform the structure of the ocean crust. These features are named in studies and publications in a disorganized way. As the study of the seabed extends, it is necessary to assign a name to them using technical considerations that have been standardized by the International Hydrographic Organization (IHO) and the Sub-Committee on Undersea Feature Names (SCUFN) for the thematic underwater toponymy. In the present work, the existence of the Galera seamount was determined through bathymetric data modelling with the Geographic Information System tool “ArcGis", which was approved by SCUFN in 2017 because it shows an elevation higher than $1000 \mathrm{~m}(1027)$ with respect to the surroundings. The geodynamic behaviour of mount Galera is of regional importance because it is estimated that it could be in the limit of the trench in $\sim 15000$ years.
\end{abstract}

Keywords - Asperities, bathymetry, seabed, seamounts.

\section{INTRODUCCIÓN}

La designación desordenada de las formas de relieve submarino, que frecuentemente aparecen en publicaciones científicas o en las cartas y mapas oceánicos, sin un estudio minucioso para conocer si el nombre asignado es apropiado o si la forma ya ha sido nombrada y descubierta, ha generado seria preocupación durante los últimos años [1], [2].

A medida que la recolección de información batimétrica a nivel mundial mejora, se descubren y definen más características geomorfológicas en los fondos oceánicos, por lo que es importante conseguir una mayor uniformidad y estandarización oficial en los nombres geográficos de las formas del relieve submarino descubiertas [2].

Con el fin de remediar esta situación, la Organización Hidrográfica Internacional (OHI), publicó varias directrices incluyendo el "Formulario de Propuesta de nombres" y la "Lista de Términos y Definiciones para la normalización de las formas del relieve submarino”, elaborados gracias a la colaboración del Subcomité de los Nombres de las Formas del Relieve Submarino de la GEBCO (SCUFN por sus siglas en inglés), y el Grupo de Expertos de las Naciones Unidas sobre los Nombres Geográficos (UNGEGN), conforme a las disposiciones de las Resoluciones de las Conferencias de las Naciones Unidas sobre los Nombres Geográficos [1].

El Comité encargado de estandarizar y acreditar la toponimia submarina, que es descubierta y reportada por diversos investigadores oceanográficos e hidrográficos en todo el mundo, es el Sub-Committee on Undersea Feature Names (SCUFN). La preparación de propuestas de nombres de formas del relieve submarino debe seguir las directrices contenidas en la publicación B6, la misma que en su sección 2 muestra los términos y definiciones de las formas de relieve submarino, considerando hasta ahora 44 términos genéricos propuestos por la SCUFN y 12 términos genéricos usados para armonización con otras definiciones y gacetillas de nombres submarinos [1].

Los países ribereños son generalmente responsables del mapeo de los espacios marítimos sobre los cuales posee soberanía como parte de los compromisos descritos en la Convención de las Naciones Unidas sobre el derecho de mar (CONVEMAR) [3]. Adicionalmente, en los últimos años, muchos países costeros han llevado a cabo ambiciosos programas de mapeo de fondo marino con el fin de extender los derechos soberanos más allá de las 200 millas náuticas, además de proyectos enmarcados en la caracterización de todos los fondos marinos como el proyecto SeaBed 2030 [4].

Por su parte, Ecuador realiza cada año un levantamiento de información batimétrica en sus espacios marítimos jurisdiccionales y en zonas aledañas que posean potencial de extensión de plataforma continental. Con la data batimétrica obtenida por el Instituto Oceanográfico de la Armada (INOCAR), es posible descubrir y sustentar los diferentes rasgos geomorfológicos que se encuentran en el fondo 
marino.

Desde el año 2010, el Ecuador a través del INOCAR ha enviado 9 propuestas para nombrar formas de relieve submarino descubiertas por personal técnico y científico ecuatoriano, cuyos términos genéricos corresponden a montes submarinos, colinas, lomas, entre otros [5]. Sin embargo, existen otros rasgos submarinos en zonas ecuatorianas que se encuentran nombrados en Gacetilla de Nombres de Formas del Relieve Submarino de GEBCO, esto ocurre debido a que al iniciarse el proceso de estandarización, la SCUFN integró información de base de datos de investigación existentes hasta ese momento.

Los nombres de las geoformas aprobadas hasta la presente fecha por la SCUFN en los espacios marítimos ecuatorianos y áreas de potencial extensión son: Amadeus, Carnegie, Galera, INOCAR, Libertad, Galápagos y Sun Ray (en la categoría de montes submarinos); Aromo, Orión y Flamingo, con dos picos (clasificados como colinas submarinas); Hancock y Pinta (clasificados como bancos); Bajo MacGowen (clasificado como reef), y Megaprint (clasificado como loma).

\section{MÉTODO}

Para sustentar la existencia de las formas de relieve submarino, se ha establecido una metodología de tres fases, como se observa en la Fig. 1. La Fase I corresponde a la identificación, posición y extensión de la morfología del rasgo, continuando con la revisión de la Gacetilla de Nombres de Formas del Relieve Submarino de GEBCO, para conocer si dicha geoforma ha sido ya propuesta, caso contrario se procede a realizar la búsqueda de información fidedigna disponible, que sustente la existencia de dicha geoforma.

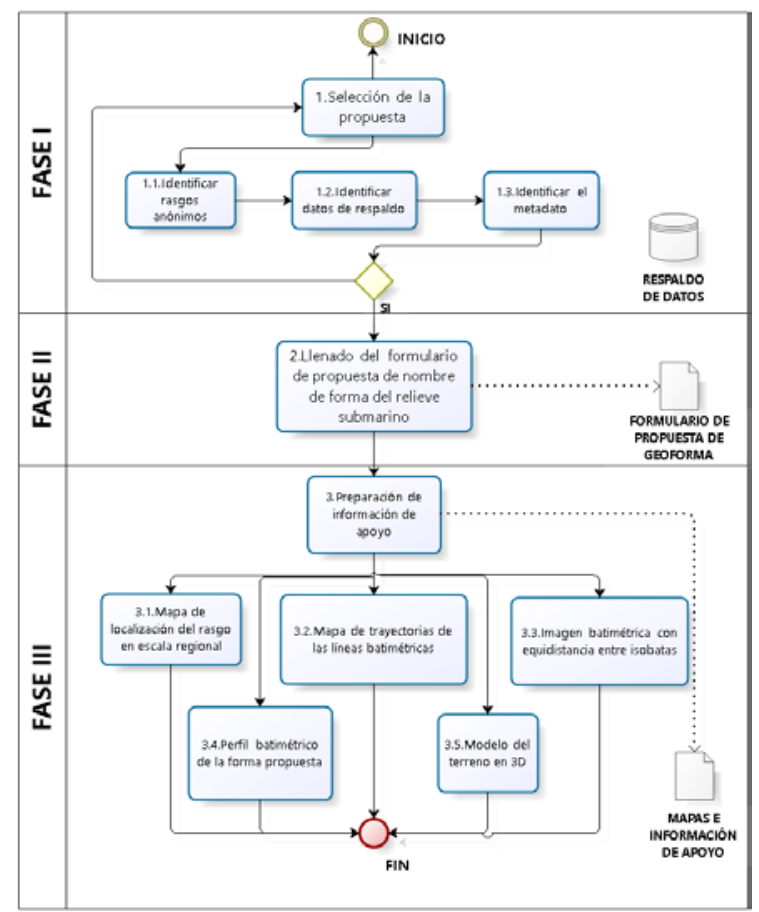

Fig. 1. Metodología para la elaboración de sustentos a fin de nombrar geoformas submarinas.

La Fase II consiste en completar el formulario proporcionado por la SCUFN, para la propuesta del rasgo submarino, siguiendo las directrices contenidas en la publicación B6 de la OHI-COI. En dicho formulario debe especificarse el nombre propuesto y la razón de la elección del nombre, coordenadas de ubicación, geometría, descripción del rasgo, referencias cartográficas, datos relativos al descubrimiento, información de los datos batimétricos de respaldo, entre otras. Existen ciertas secciones en donde será necesaria la inserción de imágenes y perfiles longitudinales o transversales que complementen la información.

La Fase III involucra la elaboración de cartografía temática que deberá suministrarse a la SCUFN para mejor apoyo de la propuesta de nombre de la forma de relieve. Esta cartografía corresponde a:

- Mapa que muestre las trayectorias de todas las líneas del levantamiento batimétrico, dentro del área donde se encuentra la geoforma.

- Imagen batimétrica donde se especifique la equidistancia entre isobatas o su índice de colores según su profundidad.

- Perfil batimétrico de la forma propuesta, indicando en una imagen la localización del perfil.

- Modelo del terreno en 3D

La información batimétrica, los formularios (en inglés) y la información de apoyo, deben enviarse a la OHI o COI, dos meses antes (en físico) o un mes antes (en digital) de la reunión anual de la SCUFN, para que esta sea considerada por sus miembros, previo a la celebración de dicha reunión [1].

\section{ANÁLISIS DE RESUltAdOS}

El monte submarino Galera fue descubierto mediante el análisis de datos batimétricos de alta resolución, obtenido en la campaña Amadeus, ejecutada en gran parte del segmento norte del margen ecuatoriano como se observa en la Fig. 2, a bordo del buque francés B/I L’Atalante en el año 2005.

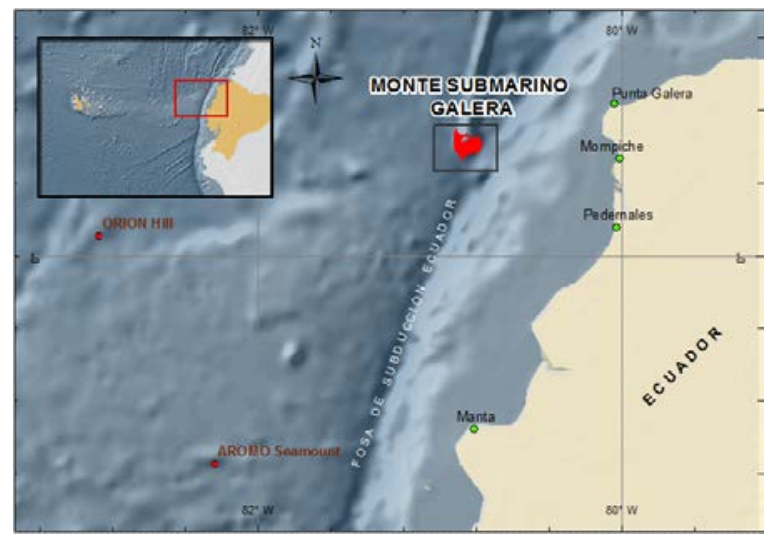

Fig. 2. Mapa de ubicación del monte submarino Galera.

Mediante el empleo de sistemas de información geográfica (SIG) y datos multihaz obtenidos en la campaña Amadeus, se elaboró un raster del modelo digital del terreno, a partir del cual se obtuvo las isobatas, el mapa de sombras y pendientes, como insumos para el análisis geomorfológico. La superficie batimétrica que encierra la geoforma en mención presentó valores que oscilaban entre los 320 y 3815 metros de profundidad. La Fig. 3 muestra un mapa batimétrico del área de estudio, en donde se observa 
que el mayor rasgo submarino encontrado corresponde a una sección de la fosa de subducción Ecuador, específicamente en el segmento central morfoestructural del margen continental ecuatoriano [6]. Desde el borde de esta fosa hacia el noreste, se observaron pequeñas protuberancias o elevaciones, una de las cuales ocupa un área aproximada de $191 \mathrm{~km}^{2}$, posee una altura de $1027 \mathrm{~m}$ y su isóbata más baja es de $3281 \mathrm{~m}$, como se muestra en el perfil longitudinal del rasgo submarino de la Fig. 3.
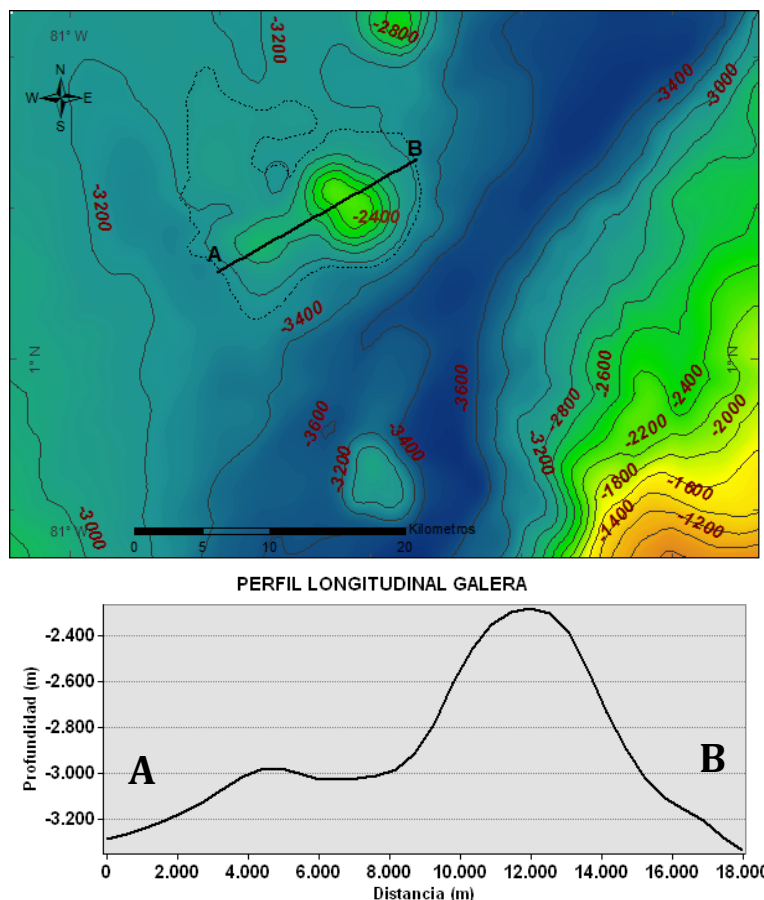

Fig. 3. Mapa batimétrico de la zona de estudio y perfil longitudinal del rasgo submarino.

Según los términos y definiciones de la publicación B-6 de la OHI-COI "Normalización de nombres de características submarinas", un monte submarino se define como "una elevación identificable generalmente equidimencional de más de $1000 \mathrm{~m}$ de altura con respecto al relieve circundante, medidos desde la isobata más profunda que la rodea en su mayor parte”. Con base en esta definición, se procedió a verificar los parámetros del rasgo submarino encontrado para corroborar su clasificación dentro de este grupo (ver Fig. 3 y Fig. 4), los datos más importantes que fueron enviados ante la SCUFN se resumen en la Tabla 1.

TABLA I

CARACTERÍSTICAS SUSTANCIALES DEL RASGO SUBMARINO

\begin{tabular}{|c|c|}
\hline Datos & Valores \\
\hline Coordenadas de Ubicación & $\begin{array}{ll}\text { Latitud } & \text { Longitud } \\
00^{\circ} 41.60^{\prime} \mathrm{N} & 80^{\circ} 54.62^{\prime} \mathrm{W}\end{array}$ \\
\hline profundidad máxima & $3281 \mathrm{~m}$ \\
\hline profundidad mínima & $2254 \mathrm{~m}$ \\
\hline Altura de la geoforma & $1027 \mathrm{~m}$ \\
\hline Área & $191 \mathrm{~km}^{2}$ \\
\hline Pendiente & $75 \%$ \\
\hline Fecha de levantamiento & Febrero/marzo 2005 \\
\hline Plataforma científica & RV L'Atalante \\
\hline Equipo de sondeo & EM12Dual \\
\hline Precisión horizontal estimada & 0.027 \\
\hline Distancia entre líneas & $3000-140000 \mathrm{~m}$ \\
\hline
\end{tabular}

Una vez identificada la geoforma y obtenida la información técnica que sustente su existencia, se propuso el nombre de Galera, debido a su cercanía con el rasgo geográfico sobresaliente conocido como Punta Galera, ubicado en el borde costero, al sur de la provincia de Esmeraldas en el Ecuador continental. (Ver Fig. 2).

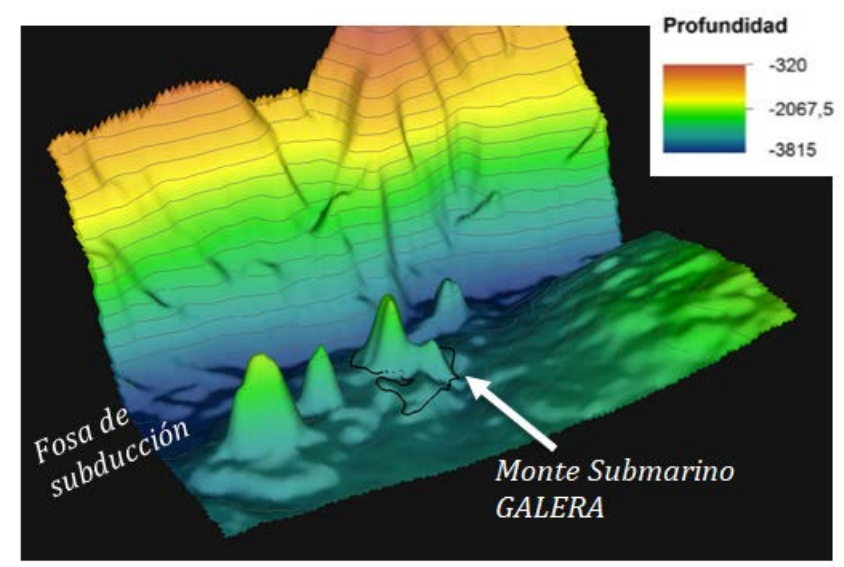

Fig. 4. Vista del modelo del terreno en 3D de la geoforma submarina Galera, donde se observa la cercanía hacia la fosa de subducción.

El monte submarino Galera está ubicado a una distancia aproximada de $9.44 \mathrm{~km}$ en el segmento norte de la fosa de subducción ecuatoriana, donde la placa de Nazca se subduce por debajo de la placa sudamericana a razón de $54 \mathrm{~mm} /$ año [8]. Debido a su proximidad y a la velocidad de subducción es posible que en 15000 años, este monte submarino se encuentre en el límite de la fosa.

El interés por el rol que desempeñan los montes submarinos está relacionado con los peligros geológicos [7]. Una vez que un monte submarino ingresa en una zona de subducción, se espera que afecte profundamente la morfología, estructura e historia del movimiento vertical de la región del antearco entre el eje de la fosa de subducción y el arco volcánico. La subducción del monte submarino también puede influir en el grado de acoplamiento entre las placas superior e inferior y puede afectar la sismicidad, especialmente la magnitud y la frecuencia de grandes terremotos [7].

Estudios recientes han demostrado que los montes submarinos juegan un papel importante controlando el historial de ruptura de grandes terremotos, en particular, actuando como barreras o como asperidades en los terremotos [9], [10].

Asimismo, la localización de las geoformas permite conocer los sitios de concentración de biodiversidad debido a los diferentes gradientes de profundidad y pendiente. Estas diferencias batimétricas conllevan variaciones en la productividad marina y en los patrones de biodiversidad asociados a la complejidad del hábitat [11].

\section{CONCLUSIONES}

El monte submarino Galera fue aprobado por la SCUFN e incluido en Undersea Feature Names Gazetteer en el año 2017, ocupando una superficie de aproximadamente 191 $\mathrm{km}^{2}$ y cuya profundidad máxima y mínima corresponde a 3281 m y 2264 m, respectivamente. Hasta 2017, se han descubierto y caracterizado un total de 15 formas del relieve submarino en el territorio marítimo ecuatoriano, 9 de ellas 
por parte del INOCAR. Sin embargo, la morfología del fondo marino del espacio marítimo ecuatoriano es muy compleja, por lo que es probable que existan diferentes formas del relieve submarino aún desconocidas. La subducción de los montes submarinos está relacionada con la sismicidad de las placas convergentes, actuando como barrera o asperidad. Futuros trabajos deberían centrarse en un estudio de Galera, mediante levantamiento sísmico, batimétrico, y aplicación de métodos directos de exploración a fin de conocer las propiedades geológicas y para determinar su papel en la sísmica de subducción.

\section{REFERENCIAS}

[1] P. Form and F. D. E. Propuesta, "Standardization of undersea feature names", Guidelines proposal form terminology. Monaco, 2017.

[2] A. J. Kerr ', "The International Hydrographic Organization and Its Involvement With Geographical Place Names,” Int. Hydrogr. Rev., no. $1,1998$.

[3] NNUU, United Nations Convention on the Law of the Sea. Spanish text, vol. 1834. 1982.

[4] L. Mayer, M. Jakobsson, G. Allen, B. Dorschel, R. Falconer, V. Ferrini, G. Lamarche, H. Snaith, and P. Weatherall, "The Nippon Foundation-GEBCO Seabed 2030 Project: The Quest to See the World's Oceans Completely Mapped by 2030,” Geosciences, vol. 8, no. 2, p. 63, 2018.

[5] I. H. O. Intergovernmental Oceanographic Commisssion (of UNESCO), "Twenty fourth meeting of the GEBCO Sub-Committee on Undersea Feature Names (SCUFN),” Beijing, China, 2011.

[6] A. P. Jean-Yves Collot, François Michaud, Alexandra Alvarado, Boris Marcaillou, Marc Sosson, Gueorgui Ratzov, "Visión general de la morfología submarina del margen convergente de EcuadorSur de Colombia: implicaciones sobre la transferencia de masa y la edad de la subducción de la Cordillera de Carnegie,” Geol. y Geofísica Mar. y Terr. del Ecuador, pp. 47-74, 2009.

[7] A. Watts, A. Koppers, and D. Robinson, "Seamount Subduction and Earthquakes,” Oceanography, vol. 23, no. 01, pp. 166-173, 2010.

[8] R. Trenkamp, J. N. Kellogg, J. T. Freymueller, and H. P. Mora, "Wide plate margin deformation, southern Central America and northwestern South America, CASA GPS observations,” J. South Am. Earth Sci., vol. 15, no. 2, pp. 157-171, 2002.

[9] A. Nishizawa, K. Kaneda, N. Watanabe, and M. Oikawa, "Seismic structure of the subducting seamounts on the trench axis: Erimo Seamount and Daiichi-Kashima Seamount, northern and southern ends of the Japan Trench,” Earth, Planets Sp., vol. 61, no. 3, pp. e5e8, 2009.

[10] S. Das and A. B. Watts, "Effect of Subducting Seafloor Topography on the Rupture Characteristics of Great Subduction Zone Earthquakes,” in Subduction Zone Geodynamics, 2009, pp. 103-118.

[11] C. R. McClain and L. Lundsten, "Assemblage structure is related to slope and depth on a deep offshore Pacific seamount chain," Mar. Ecol., vol. 36, no. 2, pp. 210-220, 2015. 